\title{
Assessment of myocardium at risk with contrast enhanced steady-state free precession cine cardiovascular magnetic resonance compared to single-photon emission computed tomography
}

\author{
Peder Sörensson', Einar Heiberg³, Nawsad Saleh', Frederic Bouvier², Kenneth Caidahl2, Per Tornvall', Lars Rydén', \\ John Pernow ${ }^{1}$ and Håkan Arheden*3
}

\begin{abstract}
Background: Final infarct size following coronary occlusion is determined by the duration of ischemia, the size of myocardium at risk (MaR) and reperfusion injury. The reference method for determining MaR, single-photon emission computed tomography (SPECT) before reperfusion, is impractical in an acute setting. The aim of the present study was to evaluate whether MaR can be determined from the contrast enhanced myocardium using steady-state free precession (SSFP) cine cardiovascular magnetic resonance (CMR) performed one week after the acute event in STelevation myocardial infarction (STEMI) patients with total coronary occlusion.

Results: Sixteen patients with STEMI (age $64 \pm 8$ years) received intravenous 99 m-Tc immediately before primary percutaneous coronary intervention. SPECT was performed within four hours. MaR was defined as the non-perfused myocardial volume derived with SPECT. CMR was performed $7.8 \pm 1.2$ days after the myocardial infarction using a protocol in which the contrast agent was administered before acquisition of short-axis SSFP cines. MaR was evaluated as the contrast enhanced myocardial volume in the cines by two blinded observers. MaR determined from the enhanced region on cine CMR correlated significantly with that derived with SPECT $\left(r^{2}=0.78, p<0.001\right)$. The difference in MaR determined by CMR and SPECT was $0.5 \pm 5.1 \%$ (mean \pm SD). The interobserver variability of contrast enhanced cine SSFP measurements was $1.6 \pm 3.7 \%$ (mean \pm SD) of the left ventricle wall volume.
\end{abstract}

Conclusions: Contrast enhanced SSFP cine CMR performed one week after acute infarction accurately depicts MaR prior to reperfusion in STEMI patients with total occlusion undergoing primary PCI. This suggests that a single CMR examination might be performed for determination of MaR and infarct size.

\section{Background}

The extent of myocardial injury following myocardial infarction (MI) is an important determinant of short and long term prognosis [1]. Early reperfusion, either by pharmacological thrombolysis or by primary percutaneous coronary intervention, is therefore mandatory for the effective myocardial salvage [2,3]. The introduction of reperfusion therapy has lead to considerable improvement in the survival of patients with acute ST-elevation MI (STEMI) [4]. Still many patients develop extensive

* Correspondence: hakan.arheden@med.lu.se

3 Lund University Hospital, Department of Clinical Physiology, Lund, Sweden Full list of author information is available at the end of the article myocardial damage underlining the need for therapeutic modalities that limit the extent of the final injury. The development of such treatment is dependent on methods that accurately determines the size of the jeopardized myocardium at risk (MaR) and that are feasible to use in patients presenting with STEMI.

MaR comprises both myocardial tissue that is irreversibly injured at the time of reperfusion and a viable border zone of reversibly injured cells [5]. The difference between MaR and infarct size is used to calculate the myocardial salvage index which is a measurement of the effectiveness of interventions that aim to reduce the extent of the final myocardial infarct [6]. The reference 
method for determining $\mathrm{MaR}$ is single photon emission computer tomography (SPECT) [7-9]. This method requires injection of a labelled isotope before reperfusion which, besides being difficult to accomplish in an acute setting, may delay the time to the coronary intervention and exposes the patient to additional radiation. These factors clearly limit the use of SPECT in clinical trials and illustrate the need for development of new methods to determine MaR.

Cardiovascular magnetic resonance (CMR) has an excellent in-plane spatial resolution, producing precise measurement of infarct size $[10,11]$ without exposing the patient to ionizing radiation. In 2005 Laissy et al. evaluated the diagnostic value of contrast enhanced (CE) timeresolved balanced steady-state free precession (SSFP) in the assessment of infarct size compared with late gadolinium enhancement (LGE) sequences [12]. They found a close correlation and concluded that "CE cine-SSFP sequences should play a role in assessing necrotic and jeopardized myocardium after acute MI". Experimental [13-15] and clinical [16-19] studies suggest that MaR can be detected with different T2-weighted CMR depicting the initial oedema several days after the acute MI. T2weighted imaging for quantification of $\mathrm{MaR}$ was recently validated in humans using myocardial perfusion SPECT [20]. Alternatively, it has been suggested that unenhanced T1-weighted images can be used to quantify oedema [21], infarcted endocardial surface area (infarct-ESA) [22] or single-shot dark blood-prepared SSFP [23] for potential subsequent identification of MaR.

We used a modified CMR protocol in which gadolinium contrast agent is injected just before SSFP-imaging is commenced for LV volumes and function, which is an important distinction from standardized methods [24]. Using this protocol we observed transmural contrast enhancement seemingly representing the $\mathrm{MaR}$ on cineSSFP sequences. This CMR protocol would thereby have the potential to determine MaR and infarct size in one single examination performed several days after the acute event if the observed transmural contrast enhancement on SSFP images represents MaR.

This study therefore tested the hypothesis that the observed contrast enhancement on cine SSFP one week after an acute MI represents MaR by comparing it with reference method myocardial perfusion SPECT obtained before opening the coronary occlusion.

\section{Materials and methods Study Protocol}

Sixteen consecutive patients, age $64 \pm 8$, with first time STEMI admitted for primary percutaneous coronary intervention (PCI) during the period February 2007 to December 2008 when myocardial perfusion scintigraphy was accessible and who fulfilled the inclusion and exclu- sion criteria were enrolled. Twelve patients were included at the Karolinska University Hospital and four at the Lund University Hospital. Myocardial perfusion isotope was mostly available during daytime, which limited the inclusion rate. Data from the four patients from Lund constitute part of an earlier published study [20]. Inclusion criteria were: chest pain $\geq 30$ minutes and $\leq 9$ hours duration, ST-elevation in at least two contiguous ECG leads or left bundle branch block and a complete coronary occlusion (TIMI flow grade 0) of the infarct-related artery at the time of coronary angiography. Exclusion criteria were prior history of MI, prior coronary artery bypass grafting, cardiogenic shock, known renal insufficiency, contraindications for CMR, permanent atrial fibrillation. The study was approved by the independent local ethics committee at each center. Written informed consent was obtained from all patients. The protocol was designed, conducted and analysed with Good Clinical Practice regulation.

\section{Coronary Angiography}

Coronary angiography was performed to confirm complete coronary occlusion in the infarct related artery and coronary angioplasty was then performed according to local standard procedures at the discretion of the individual physician. The intervention was completed by a coronary angiogram to determine final TIMI grade flow.

\section{SPECT}

Prior to opening of the occluded vessel the patients received a body weight-adjusted (350-700 MBq) iv injection of 99 mTc tetrofosmin (Amersham Health, Buckinghamshire, UK) or sestamibi (MIBI, Cardio-lite, Bristol Myers Squibb, USA). Myocardial perfusion SPECT imaging was performed within four hours to visualize and quantify MaR using either of two dual-head cameras: GE camera (Ventri, GE Healthcare) or Sopha camera (DSTXL; Sopha Medical Vision, Bue Cedex, France). The patients were placed in the supine position and imaged in steps of 5.6 degrees using a $64 \times 64$ matrix, with a typical pixel size of $5 \times 5 \mathrm{~mm}$ and a slice thickness of $5 \mathrm{~mm}$. The reconstructed voxel size was $3 \times 3 \times 3 \mathrm{~mm}$ (Sopha) or 6.4 $\times 6.4 \times 6.4 \mathrm{~mm}(\mathrm{GE})$. Image acquisition time was approximately $15 \mathrm{~min}$. Iterative reconstruction using maximumlikelihood expectation maximization was performed with a low-resolution Butterworth filter and a cut off frequency set to 0.5 of Nyquist and an order of 5.0. No attenuation or scatter correction was applied and short-axis images were reconstructed semi-automatically on the respective workstation for each camera.

\section{CMR}

A standard clinical CMR protocol, except for the time of administration of contrast, was scheduled to be performed one week after the onset of symptoms. Timing 
was chosen at one week to avoid the early infarct phase where a rapid decrease in infarct size has been reported [25]. Two $1.5 \mathrm{~T}$ systems were used: Signa Excite TwinSpeed (General Electric Healthcare, Waukesha, WI, USA) or Philips Intera CV (Philips, Best, Nederlands). Eight(GE) and five-channel (Philips) cardiac-coil was used and all patients were in the supine position with vector-ECG monitoring. A bolus of gadolinium contrast agent $(0.2$ $\mathrm{mmol} / \mathrm{kg}$ bodyweight (Omniscan, GE Healthcare, Norway or Magnevist, Bayer Pharma, Berlin, Germany) was given iv just before positioning the patient in the scanner. The image protocol included scout images, localization of the short axis and then covering the whole left ventricle (LV) with retrospectively gated SSFP cines. The following typical parameters on GE-scanner was used; SSFP (TE $1.58 \mathrm{~ms}$, TR $3.61 \mathrm{~ms}$, flip angle 60 degrees, 25 phases, 8 mm slice, no gap, matrix $226 \times 226$ ). LGE images were acquired 15-20 minutes after contrast injection using an inversion recovery gradient echo sequence (TE $3.3 \mathrm{~ms}$, TR $7.0 \mathrm{~ms}$, TI 180-250 ms to null the myocardium, $8 \mathrm{~mm}$ slice, no gap, matrix $256 \times 192)$ and the same slice orientation as cine SSFP images. Typical parameters on the Philips scanner was; SSFP (TE $1.4 \mathrm{~ms}$, TR $2.8 \mathrm{~ms}$, flip angle 60 degrees, 30 phases, $8 \mathrm{~mm}$ slice, matrix $160 \times$ 141). LGE images were acquired 15-20 minutes after contrast injection using inversion recovery gradient echo sequence (TE $1.14 \mathrm{~ms}$, TR, $3.8 \mathrm{~ms}$, TI $180-250 \mathrm{~ms}, 8 \mathrm{~mm}$ slice, no gap, matrix $240 \times 180$ ). Cardiac triggering was set for diastole to reduce motion artefacts. Each slice was obtained during end-expiratory breath holding. Two-, three- and four chamber views were also obtained to confirm the findings.

\section{Image analysis and evaluation}

Analysis of myocardial perfusion SPECT defect for MaR was performed off-line using freely available segmentation software (Segment v1.702; http://segment.heiberg.se)[26,27]. The automatic segmentation finds the centerline through the left ventricular wall and identifies the endo- and epicardium based on an individually estimated wall thickness and signal intensity values within the image [28]. Manual adjustment of the automatic delineation was sometimes required in the left ventricular outflow region. The perfusion defect was determined by an automated algorithm that considers myocardium with $<55 \%$ of normal counts as being ischemic [29]. MaR was quantified as \% of the left ventricle.

CMR images were analysed off-line using the same software Segment. End-diastolic and end-systolic volumes, ejection fraction, stroke volume and left ventricular volume was calculated on SSFP cines. The contrast enhanced myocardial volume in SSFP cines was manually delineated in end-diastole and end- systole by two observers blinded to each other's findings and to SPECT results. The values were averaged for internal control and expressed as \% of the LV wall volume. See additional file 1: Movie 1 for the original data used to perform this analysis. The relatively low contrast difference between contrast in injured myocardium and remote myocardium precluded the use of a semi-quantification algorithm. Infarction was quantified, in a later session, using an automated quantification method [30] that has been validated in ex vivo and in vivo experiment in which partial volume effects are accounted for.

To determine the image relative intensity ratio between enhanced and remote myocardium a region of interest (ROI) was manually drawn in approximately $80 \%$ of the enhanced area in three consecutive slices and the same size ROI was drawn in the remote myocardium.

\section{Cardiac enzymes}

Cardiac biomarkers were sampled every fourth hour after admission on day one and every sixth hour on day two. Troponin-T was analysed with immunoassay technique (Modular Analytics E-module, Roche Diagnostics) and CK-MB was analysed using chemical luminescence technique (UniCel DxI 800, Beckman Coulter AB).

\section{Statistics}

All data are expressed as mean and standard deviation. Wilcoxon Mann-Whitney Rank Sum test was used to test for differences between groups (SPECT and contrast enhanced SSFP). Wilcoxon Sign Rank test was used to test the relative signal enhancement in contrast enhanced regions on SSFP images compared to remote myocardium. Bland-Altman plots were constructed for comparing contrast enhanced cine SSFP images with reference standard myocardial perfusion SPECT. Interobserver variability was calculated as the standard deviation of the difference between two blinded observers divided by the average of the two observers. A two-sided P-value $<0.05$ was considered statistically significant. Statistical analysis was performed using commercially available software GraphPad Prism version 5.00 for Windows (GraphPad Software, San Diego, CA, USA, http://www.graphpad.com). The authors had full access to the data and take responsibility for its integrity. All authors have read and agreed to the manuscript as written.

\section{Results \\ Patients}

The culprit lesion was located in the right coronary artery (RCA) in twelve patients, in the left anterior descending artery (LAD) in two and in the left circumflex artery $(\mathrm{LCx})$ in one (Table 1). All patients received oral antiplatelet therapy with loading doses of $320-500 \mathrm{mg}$ aspirin and 300 or $600 \mathrm{mg}$ clopidogrel before the intervention and iv infusion of a GPIIb/IIIa inhibitor in connection 
Table 1: Myocardium at risk comparing SPECT and CMR. Infarct size measured with LGE and myocardial salvage index

\begin{tabular}{|c|c|c|c|c|c|}
\hline & Culprit lesion & $\begin{array}{l}\text { MaR SPECT } \\
\text { (LV\%) }\end{array}$ & MaR CMR (LV\%) & $\begin{array}{l}\text { Infarct size LGE } \\
\text { (LV\%) }\end{array}$ & $\begin{array}{l}\text { Salvage Index } \\
\text { (\%) }\end{array}$ \\
\hline Patient 1 & $\mathrm{RCA}$ & 38 & 31 & 15 & 52 \\
\hline Patient 2 & LAD & 51 & 47 & 30 & 36 \\
\hline Patient 3 & $\mathrm{RCA}$ & 30 & 35 & 1 & 97 \\
\hline Patient 4 & $\mathrm{RCA}$ & 30 & 30 & 9 & 70 \\
\hline Patient 5 & $\mathrm{RCA}$ & 31 & 28 & 14 & 50 \\
\hline Patient 6 & $\mathrm{RCA}$ & 15 & 23 & 2 & 91 \\
\hline Patient 7 & LAD & 37 & 32 & 11 & 66 \\
\hline Patient 8 & $\mathrm{RCA}$ & 26 & 24 & 9 & 63 \\
\hline Patient 9 & $\mathrm{RCA}$ & 25 & 21 & 9 & 57 \\
\hline Patient 10 & $\mathrm{RCA}$ & 28 & 29 & 7 & 76 \\
\hline Patient 11 & $\mathrm{RCA}$ & 11 & 17 & 10 & 41 \\
\hline Patient 12 & $\mathrm{RCA}$ & 26 & 24 & 10 & 58 \\
\hline Patient 13 & $\mathrm{RCA}$ & 14 & 23 & 5 & 78 \\
\hline Patient 14 & $\mathrm{RCA}$ & 18 & 24 & 9 & 63 \\
\hline Patient 15 & $C X$ & 28 & 27 & 9 & 67 \\
\hline Patient 16 & RCA & 22 & 27 & 1 & 96 \\
\hline
\end{tabular}

$\mathrm{RCA}=$ right coronary artery; $\mathrm{LAD}=$ left ascending artery; $\mathrm{CX}=$ left circumflex artery;

$\mathrm{MaR}=$ Myocardium at Risk; SPECT $=$ single-photon emission computed tomography;

$\mathrm{CMR}=$ cardiovascular magnetic resonance; $\mathrm{LV}=$ left ventricle. Myocardial salvage index

(1-scar/MaR).

A

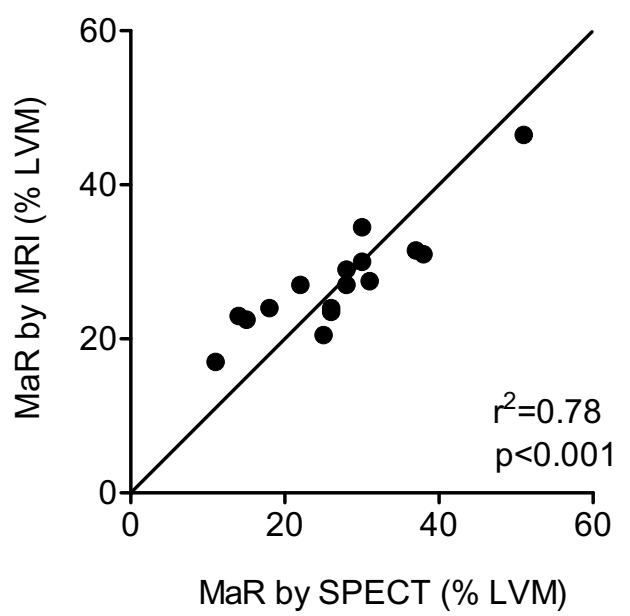

\section{B}

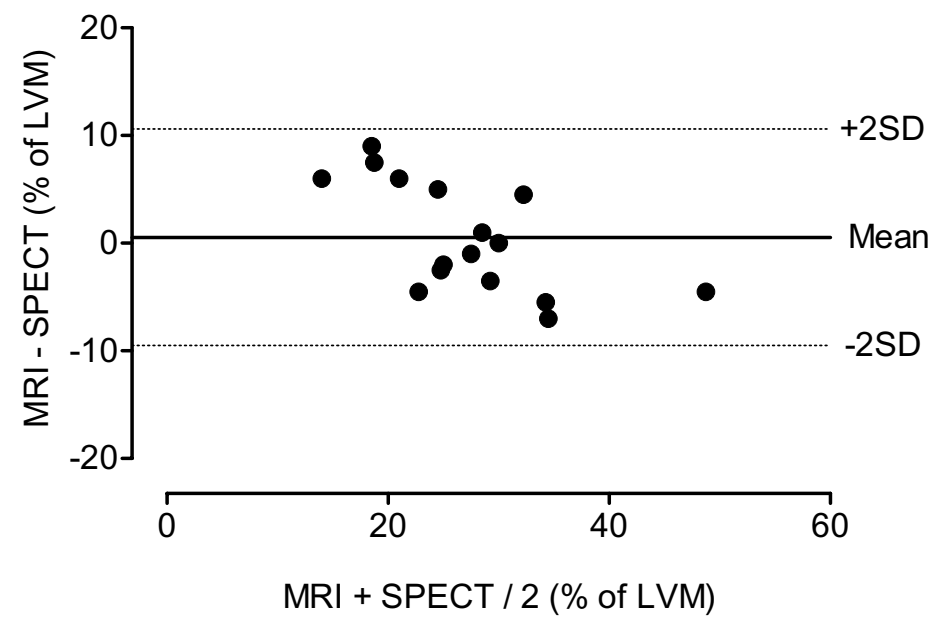

Figure 1 Agreement between MaR determined by CMR and SPECT. Panel A: Scatter plot showing MaR one week after reperfusion determined by gadolinum enhanced SSFP cines plotted versus MaR as it was before reperfusion determined by myocardial perfusion SPECT together with best line and line of identity. Panel B: Bland-Altman plot showing the agreement between MaR determined by myocardial perfusion SPECT and contrast enhanced cine SSFP. The difference was $0.5 \pm 10 \%$ (mean \pm 2 SD). 
with the PCI procedure. TIMI flow grade 3 was achieved in the affected artery in all patients. The time from onset of chest pain to reperfusion was $222 \pm 120$ minutes. The maximum levels of troponin- $\mathrm{T}$ and CK-MB were 7.0 \pm 5.6 $\mu \mathrm{g} / \mathrm{l}$ and $252 \pm 210 \mu \mathrm{g} / \mathrm{l}$, respectively. There were no severe adverse events or reinfarctions prior to the CMR investigation that was performed $7.8 \pm 1.2$ days after the onset of symptoms.

\section{CMR \& SPECT}

Images of good diagnostic quality were obtained in all patients. Throughout the entire RR-interval a high transmural signal was consistently observed in the infarct region, and the infarct was always within the area of contrast enhancement in the SSFP cines. MaR, defined as the non-perfused myocardial volume on SPECT, ranged from 11 to $51 \%$ (mean $27 \pm 10 \%$ ) of the LV wall volume. The contrast enhanced region on SSFP cines, calculated as the mean values obtained at end-diastole and end-systole, ranged from 17 to $47 \%$ (mean $27 \pm 7 \%$ ) of the $\mathrm{LV}$ wall volume. As illustrated in Figure 1A there was a good correlation between $\mathrm{MaR}$ determined from the enhanced region on SSFP cines and that determined with SPECT $\left(\mathrm{r}^{2}=\right.$ $0.78, \mathrm{p}<0.001$ ). The difference between the enhanced region on SSFP cines and MaR on SPECT was $0.5 \pm 5.1 \%$ $(\mathrm{p}=0.60$, Figure $1 \mathrm{~B})$. The location of the enhanced region on SSFP cines always agreed with MaR on myocardial perfusion SPECT images. Two typical examples of MaR and infarct area in the RCA and LAD regions are shown in Figures 2 and 3. The signal intensity ratio between regions of gadolinium enhanced and remote myocardium

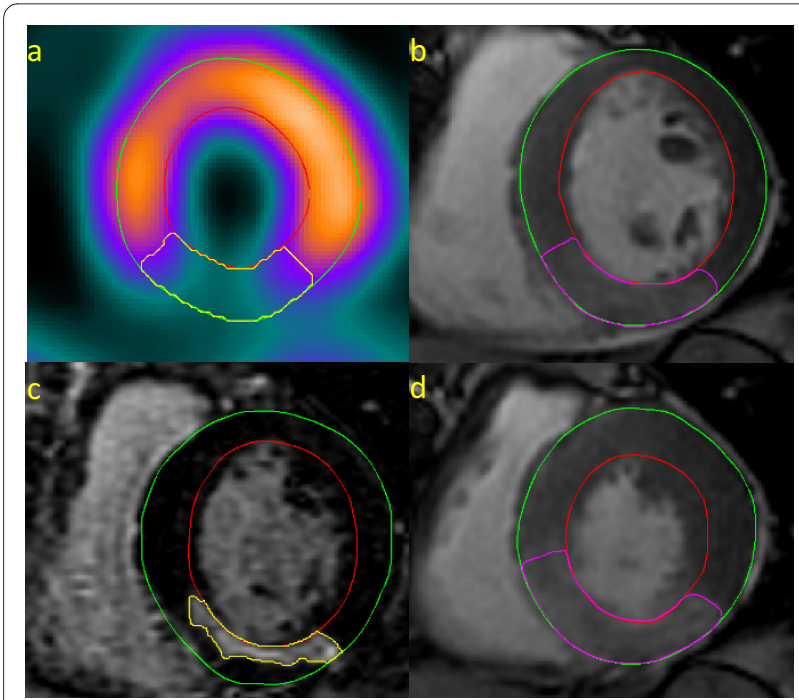

Figure 2 Inferior STEMI. Corresponding left ventricular short axis views from a patient with inferior STEMI. MaR determined by (a) myocardial perfusion SPECT, (b) gadolinium enhanced SSFP at end-diastole, (c) infarct size images with LGE and (d) gadolinium enhanced SSFP at end-systole. was $1.42 \pm 0.25(\mathrm{p}<0.001)$. The interobserver variability for gadolinium enhanced myocardium between two readers was $1.6 \pm 3.7 \%$. Infarct size determined by CMR ranged from 1 to $30 \%$ (mean $9 \pm 7 \%$ ) of LV wall volume and mean transmurality ranged from 26 to $52 \%$.

\section{Discussion}

This study demonstrates that the contrast enhanced myocardium on SSFP cines represents the MaR as determined by myocardial perfusion SPECT. Thus gadolinium enhanced CMR performed one week after an acute STEMI can accurately determine MaR simultaneously with the infarct size.

The extent of the final infarct size is dependent on several factors including the duration of ischemia, the degree of collateral flow, myocardial oxygen demand and the size of MaR [1]. In studies aimed at limiting infarct size, accurate determination of $\mathrm{MaR}$ is crucial in order to calculate the myocardial salvage index (Figure 4). The reference standard method for determination of MaR is SPECT which has the important limitation that the isotope needs to be prepared and injected before the ischemic myocardium is reperfused. Since isotope for SPECT may not be readily available in the acute setting of primary $\mathrm{PCI}$, new methods which are more feasible to use in patients with acute MI need to be developed.

Our results differ from those of an earlier study in 2005 where there was a good correlation between gadolinium

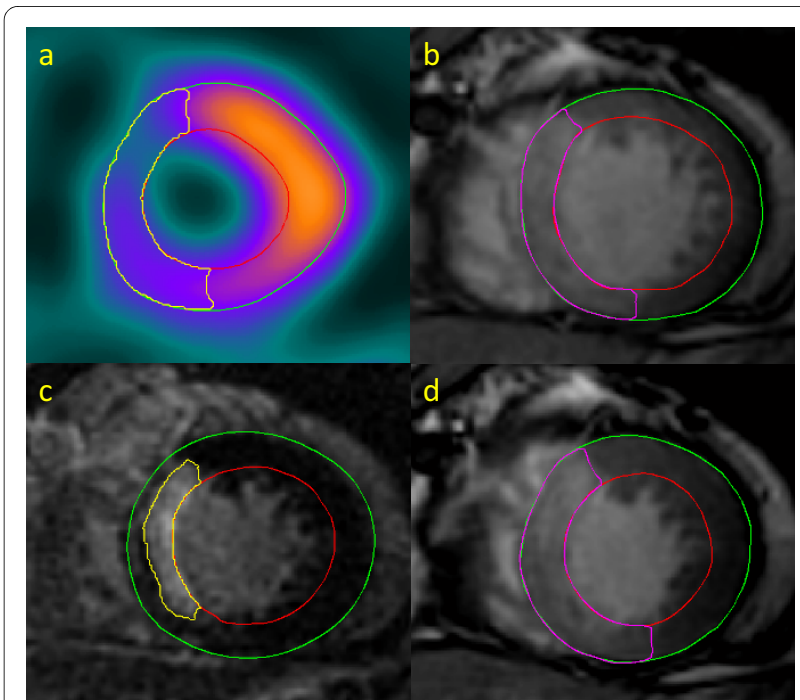

Figure 3 Anterior STEMI. Corresponding left ventricular short axis views from a patient with anterior myocardial STEMI. MaR determined by (a) myocardial perfusion SPECT, (b) gadolinium enhanced SSFP at end-diastole, (c) infarct size images with LGE and (d) gadolinium enhanced SSFP at end-systole. It is clearly seen that the region of gadolinium enhancement does not correspond in size or endocardial extent to the region of myocardium at risk either by SSFP cines or myocardial SPECT. 

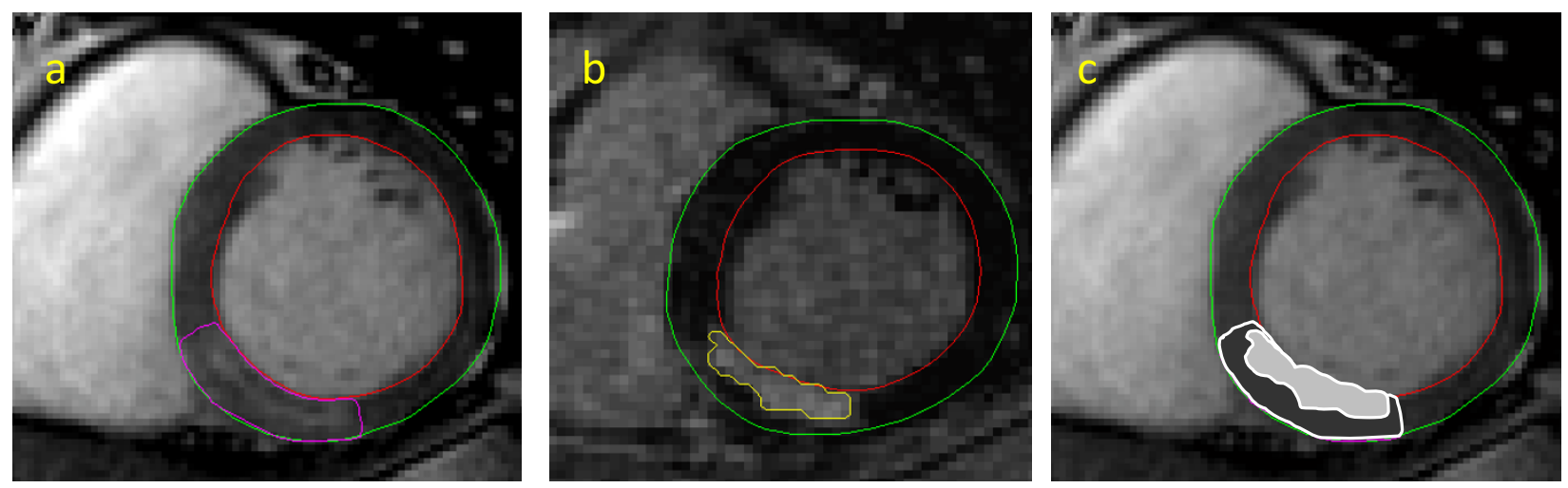

Figure 4 Myocardial salvage. Corresponding left ventricular short axis views from a patient with inferior STEMI. MaR determined by (a) gadolinium enhanced SSFP at end-diastole, (b) infarct size images with LGE and (c). The infarcted area from panel b is superimposed on MaR from panel a (light grey shade on dark striped area). Myocardial salvage index is calculated as (1-scar/MaR).

SSFP cines and infarct size on LGE [12]. These differences may in part be attributable to differences in study populations with less transmural enhancement on gadolinium SSFP cines in the present study likely due to shorter pain-to-opening times Another possible explanation may be that our study included only STEMI patients.

Previous attempts to investigate MaR through CMR focused on different T2-weighted sequences. It was suggested that MaR can be estimated using T2-weighted imaging with short inversion time inversion-recovery (STIR) or T2-prepared single-shot SSFP or a combination of both $[13,17,18,22]$. The mechanism is not fully known but oedema caused by the transient ischemia, cellular swelling and impaired microvascular reperfusion have been suggested [31]. T2-weighted imaging to quantify $\mathrm{MaR}$ was first validated in humans using myocardial perfusion SPECT as reference [20]. The $\mathrm{ACUT}_{2} \mathrm{E}$ TSE-SSFP study recently showed promising results by using a hybrid method of T2-weighting with bright-blood contrast in dogs [15].

The presently described technique also has the advantage of sequentially determining MaR and the final infarct size with excellent spatial resolution in one single examination. Since SSFP cines are available on all CMR-scanners, MaR assessed by gadolinium SSFP cines might be used as a robust independent complement in cases where T2-weighted imaging may be difficult or subjected to artefact problems. Fewer acquisitions would be required since the volumetric dataset for MaR would be acquired during the normal cine acquisitions for cardiac mass and function.

The mechanism behind the enhanced myocardium observed using gadolinium SSFP cines and the gadolinium kinetics is not clarified by the present investigation and needs to be investigated in further studies. The balanced cine SSFP sequence is known to generate T2/T1weighting. Bright signal is due to short T1- or long T2- relaxation or a combination of the two. Gadolinium based contrast agents such as Gd-DTPA increases the relaxation rates by approximately the same amount. On a percentage basis, Gd-DTPA alters the T1-relaxation to a much larger extent than T2-relaxation, since T1 in tissue is much longer than T2 [32]. With an increased concentration of contrast medium in the MaR, an increase in signal would be seen from early balanced cine SSFP echoes (i.e. proton spins that have not experienced T2relaxation), while signal originating from subsequent echoes (i.e. proton spins that have experienced T2-relaxation) would remain approximately constant. As seen in the studies using T2-weighted sequences to estimate $\mathrm{MaR}$, the bright signal seen in MaR is largely due to the prolonged T2-relaxation of the oedema. This is most likely the case using balanced cine SSFP as well, but the signal is "boosted" by the shortening of T1-relaxation in the MaR.

The present results are of potential clinical and scientific importance because it provides an easily accessible technique for quantification of the efficacy of reperfusion therapy by calculation of the myocardial salvage index [33]. The estimation of MaR and final infarct size might be achieved in a stable patient situation several days after the acute MI. This is in contrast to SPECT which requires preparation and injection of isotope before reperfusion and image acquisition within a few hours in an unstable patient.

\section{Limitations}

The low number of patients may be seen as a limitation to this study. On the other hand the material includes a large span of myocardium at risk ranging from $11-51 \%$ of the LV wall volume, which is important for the evaluation. We did not use a semi-quantitative method to determine the contrast enhanced myocardium on SSFP cines due to the relatively low signal increase compared to remote 
myocardium. No comparison was made to T2-weighted sequences because of low number of patients and that it was not the objective with this study. Other limitations are that only four out of sixteen patients were female, most infarctions involved the RCA territory and only total coronary occlusions (TIMI flow grade 0 ) were included.

\section{Conclusions}

Contrast enhanced SSFP cines performed one week after the acute event accurately depicts MaR as it was before reperfusion in STEMI patients with total occlusion undergoing primary PCI. A single CMR examination can thereby be performed for determination of $\mathrm{MaR}$ and infarct size.

\section{Additional material}

\section{Additional file 1 An example of gadolinium enhanced SSFP cine of} myocardium at risk in an inferior infarction one week after admission. The inferior midventricular wall enhancement represents MaR which can be seen both in end-diastole and end-systole. Normal myocardial signal in anterior, septal and lateral walls.

\section{Competing interests}

The authors declare that they have no competing interests.

\section{Authors' contributions}

PS enrolled the majority of the patients. PS, EH and HA participated in the design of the study and coordination, performed the statistical analysis and drafted the manuscript. NS, FB, KC, PT, LR, JP participated in the design of the study and coordination and helped to draft the manuscript. All authors read and approved the final manuscript.

\section{Acknowledgements}

The authors greatly acknowledge Kerstin Höglund, Yords österman, Marie Lundberg, Dianna Bone, Ann-Helen Arvidsson and Christel Carlander for excellent technical assistance.

Founding was received from the Swedish Heart and Lung Foundation, the Swedish Research Council $(10857,14231)$, the Stockholm County Council, the Karolinska Institute, Stockholm (JP, NS) and the Medical Faculty at Lund University $(\mathrm{HA})$

\section{Author Details}

1Department of Medicine, Karolinska Institutet, Karolinska University Hospital, Stockholm, Sweden, 2Molecular Medicine and Surgery, Karolinska Institutet, Karolinska University Hospital, Stockholm, Sweden and ${ }^{3}$ Lund University Hospital, Department of Clinical Physiology, Lund, Sweden

Received: 12 December 2009 Accepted: 30 April 2010 Published: 30 April 2010

\section{References}

1. Braunwald E: Myocardial reperfusion, limitation of infarct size, reduction of left ventricular dysfunction, and improved survival. Should the paradigm be expanded? Circulation 1989, 79:441-444.

2. Indications for fibrinolytic therapy in suspected acute myocardial infarction: collaborative overview of early mortality and major morbidity results from all randomised trials of more than 1000 patients. Fibrinolytic Therapy Trialists' (FTT) Collaborative Group. Lancet 1994, 343:311-322.

3. Kloner RA, Rezkalla SH: Cardiac protection during acute myocardial infarction: where do we stand in 2004? J Am Coll Cardiol 2004, 44:276-286
4. Antman EM, Hand M, Armstrong PW, Bates ER, Green LA, Halasyamani LK, Hochman JS, Krumholz HM, Lamas GA, Mullany CJ, Pearle DL, Sloan MA, Smith SC Jr, Anbe DT, Kushner FG, Ornato JP, Pearle DL, Sloan MA, Jacobs AK, Adams CD, Anderson JL, Buller CE, Creager MA, Ettinger SM, Halperin $J$, Hunt SA, Lytle BW, Nishimura R, Page RL, Riegel B, Tarkington LG, Yancy CW: 2007 focused update of the ACC/AHA 2004 guidelines for the management of patients with ST-elevation myocardial infarction: a report of the American College of Cardiology/American Heart Association Task Force on Practice Guidelines. J Am Coll Cardiol 2008, 51:210-247

5. Reimer KA, Jennings RB: The "wavefront phenomenon" of myocardial ischemic cell death. II. Transmural progression of necrosis within the framework of ischemic bed size (myocardium at risk) and collateral flow. Lab Invest 1979, 40:633-644.

6. Wang QD, Pernow J, Sjoquist PO, Ryden L: Pharmacological possibilities for protection against myocardial reperfusion injury. Cardiovasc Res 2002, 55:25-37.

7. Gibbons RJ, Verani MS, Behrenbeck T, Pellikka PA, O'Connor MK Mahmarian JJ, Chesebro JH, Wackers FJ: Feasibility of tomographic 99 mTc-hexakis-2-methoxy-2-methylpropyl-isonitrile imaging for the assessment of myocardial area at risk and the effect of treatment in acute myocardial infarction. Circulation 1989, 80:1277-1286.

8. De Coster PM, Wijns W, Cauwe F, Robert A, Beckers C, Melin JA: Area-atrisk determination by technetium- 99 m-hexakis-2-methoxyisobutyl isonitrile in experimental reperfused myocardial infarction. Circulation 1990, 82:2152-2162

9. Sinusas AJ, Trautman KA, Bergin JD, Watson DD, Ruiz M, Smith WH, Beller GA: Quantification of area at risk during coronary occlusion and degree of myocardial salvage after reperfusion with technetium-99 m methoxyisobutyl isonitrile. Circulation 1990, 82:1424-1437.

10. Gibbons RJ, Valeti US, Araoz PA, Jaffe AS: The quantification of infarct size. J Am Coll Cardiol 2004, 44:1533-1542.

11. Carlsson M, Arheden $H$, Higgins CB, Saeed M: Magnetic resonance imaging as a potential gold standard for infarct quantification. $J$ Electrocardiol 2008, 41:614-620.

12. Laissy JP, Hyafil F, Huart V, Serfaty JM, Chillon S, Schouman-Claeys E, Faraggi M: Value of contrast-enhanced, balanced cine-MR sequences in the assessment of apparent infarct size after acute myocardial infarction: a prospective comparison with delayed-enhancement sequences. J Magn Reson Imaging 2005, 22:765-771.

13. Aletras AH, Tilak GS, Natanzon A, Hsu LY, Gonzalez FM, Hoyt RF Jr, Arai AE: Retrospective determination of the area at risk for reperfused acute myocardial infarction with T2-weighted cardiac magnetic resonance imaging: histopathological and displacement encoding with stimulated echoes (DENSE) functional validations. Circulation 2006, 113:1865-1870

14. Tilak GS, Hsu LY, Hoyt RF Jr, Arai AE, Aletras AH: In vivo T2-weighted magnetic resonance imaging can accurately determine the ischemic area at risk for 2-day-old nonreperfused myocardial infarction. Invest Radiol 2008, 43:7-15.

15. Aletras AH, Kellman P, Derbyshire JA, Arai AE: ACUT2E TSE-SSFP: a hybrid method for T2-weighted imaging of edema in the heart. Magn Reson Med 2008, 59:229-235.

16. Abdel-Aty H, Zagrosek A, Schulz-Menger J, Taylor AJ, Messroghli D, Kumar A, Gross M, Dietz R, Friedrich MG: Delayed enhancement and T2weighted cardiovascular magnetic resonance imaging differentiate acute from chronic myocardial infarction. Circulation 2004, 109:2411-2416.

17. Kellman P, Aletras AH, Mancini C, McVeigh ER, Arai AE: T2-prepared SSFP improves diagnostic confidence in edema imaging in acute myocardial infarction compared to turbo spin echo. Magn Reson Med 2007, 57:891-897

18. Friedrich MG, Abdel-Aty H, Taylor A, Schulz-Menger J, Messroghli D, Dietz $R$ : The salvaged area at risk in reperfused acute myocardial infarction as visualized by cardiovascular magnetic resonance. J Am Coll Cardiol 2008, 51:1581-1587

19. O'Regan DP, Ahmed R, Karunanithy N, Neuwirth C, Tan Y, Durighel G, Hajnal JV, Nadra I, Corbett SJ, Cook SA: Reperfusion hemorrhage following acute myocardial infarction: assessment with T2* mapping and effect on measuring the area at risk. Radiology 2009, 250:916-922.

20. Carlsson M, Ubachs JF, Hedstrom E, Heiberg E, Jovinge $S$, Arheden $H$ Myocardium at risk after acute infarction in humans on cardiac 
magnetic resonance: quantitative assessment during follow-up and validation with single-photon emission computed tomography. JACC Cardiovasc Imaging 2009, 2:569-576.

21. Goldfarb JW, Arnold S, Han J: Recent myocardial infarction: assessment with unenhanced T1-weighted MR imaging. Radiology 2007, 245:245-250.

22. Ortiz-Perez JT, Meyers SN, Lee DC, Kansal P, Klocke FJ, Holly TA, Davidson CJ, Bonow RO, Wu E: Angiographic estimates of myocardium at risk during acute myocardial infarction: validation study using cardiac magnetic resonance imaging. Eur Heart J 2007, 28:1750-1758.

23. Green JD, Clarke JR, Flewitt JA, Friedrich MG: Single-shot steady-state free precession can detect myocardial edema in patients: a feasibility study. J Magn Reson Imaging 2009, 30:690-695.

24. Kramer CM, Barkhausen J, Flamm SD, Kim RJ, Nagel E: Society for Cardiovascular Magnetic Resonance Board of Trustees Task Force on Standardized Protocols: Standardized cardiovascular magnetic resonance imaging (CMR) protocols, society for cardiovascular magnetic resonance: board of trustees task force on standardized protocols. J Cardiovasc Magn Reson 2008, 10:35.

25. Engblom H, Hedstrom E, Heiberg E, Wagner GS, Pahlm O, Arheden H: Rapid initial reduction of hyperenhanced myocardium after reperfused first myocardial infarction suggests recovery of the periinfarction zone: one-year follow-up by MRI. Circ Cardiovasc Imaging 2009, 2:47-55

26. Heiberg E, Sjögren J, Ugander M, Carlsson M, Engblom H, Arheden H: Design and Validation of Segment - a Freely Available Software for Cardiovascular Image Analysis. BMC Medical Imaging 2010, 10:1

27. Soneson H, Engblom H, Arheden H, Heiberg E: Automatic quantification of myocardial perfusion SPECT defects in patients with acute coronary occlusion. Journal of Nuclear Cardiology 2010 in press.

28. Soneson H, Ubachs JF, Ugander M, Arheden H, Heiberg E: An Improved Method for Automatic Segmentation of the Left Ventricle in Myocardial Perfusion SPECT. J Nucl Med 2009, 50:205-213.

29. Ugander M, Soneson H, Heiberg E, Engblom H, Pals Jvd, Erlinge D, Arheden $\mathrm{H}$ : A novel method for quantifying myocardial perfusion SPECT defect size by co-registration and fusion with MRI - an experimental ex vivo imaging pig heart study. XCardiovascular Spring Meeting. Malmö 2008

30. Heiberg E, Ugander M, Engblom H, Gotberg M, Olivecrona GK, Erlinge D, Arheden $\mathrm{H}$ : Automated quantification of myocardial infarction from MR images by accounting for partial volume effects: animal, phantom, and human study. Radiology 2008, 246:581-588.

31. Phrommintikul A, Abdel-Aty H, Schulz-Menger J, Friedrich MG, Taylor AJ: Acute oedema in the evaluation of microvascular reperfusion and myocardial salvage in reperfused myocardial infarction with cardiac magnetic resonance imaging. Eur J Radiol 2009 in press.

32. Caravan P: Strategies for increasing the sensitivity of gadolinium based MRI contrast agents. Chem Soc Rev 2006, 35:512-523.

33. Schomig A, Ndrepepa G, Mehilli J, Schwaiger M, Schuhlen H, Nekolla S, Pache J, Martinoff S, Bollwein H, Kastrati A: Therapy-dependent influence of time-to-treatment interval on myocardial salvage in patients with acute myocardial infarction treated with coronary artery stenting or thrombolysis. Circulation 2003, 108:1084-1088.

doi: $10.1186 / 1532-429 X-12-25$

Cite this article as: Sörensson et al., Assessment of myocardium at risk with contrast enhanced steady-state free precession cine cardiovascular magnetic resonance compared to single-photon emission computed tomography Journal of Cardiovascular Magnetic Resonance 2010, 12:25 